\title{
Interobserver Variation of the Bolus-and-Burst Method for Pancreatic Perfusion with Dynamic - Contrast-Enhanced Ultrasound
}

\section{(ㄷ) (1) ()ㅇㅇ}

Authors

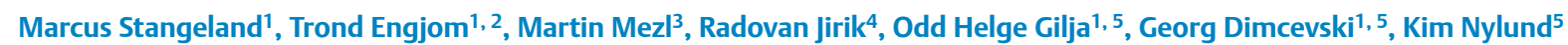

Affiliations

1 Department of Clinical Medicine, University of Bergen, Bergen, Norway

2 Department of Medicine, Haukeland Universitetssjukehus, Bergen, Norway

3 Dept. of Biomedical Engineering, Brno Univ. of Technology, Brno, Czech Republic

4 Academy of Sciences of the Czech Republic, Institute of Scientific Instruments, Brno, Czech Republic

5 National Center of Ultrasound in Gastroenterology, Haukeland University Hospital, Bergen, Norway

Key words

interobserver, dynamic contrast-enhanced ultrasound, perfusion, pancreas

received 22.10 .2016

revised 15.03.2017

accepted 19.04.2017

Bibliography

DOI https://doi.org/10.1055/s-0043-110475

Ultrasound Int Open 2017; 3: E99-E106

(C) Georg Thieme Verlag KG Stuttgart · New York

ISSN 2199-7152

Correspondence

Dr. Kim Nylund

National Center of Ultrasound in Gastroenterology

Haukeland University Hospital

Jonas Lies vei 65

5021, Bergen

Norway

Tel: + 4755972961

kim.nylund@med.uib.no

\section{ABSTRACT}

Purpose Dynamic contrast-enhanced ultrasound (DCE-US) can be used for calculating organ perfusion. By combining bolus injection with burst replenishment, the actual mean transit time (MTT) can be estimated. Blood volume (BV) can be obtained by scaling the data to a vessel on the imaging plane. The study aim was to test interobserver agreement for repeated recordings using the same ultrasound scanner and agreement between results on two different scanner systems.

Materials and Methods Ten patients under evaluation for exocrine pancreatic failure were included. Each patient was scanned two times on a GE Logiq E9 scanner, by two different observers, and once on a Philips IU22 scanner, after a bolus of $1.5 \mathrm{ml}$ Sonovue. A 60 -second recording of contrast enhancement was performed before the burst and the scan continued for another $30 \mathrm{~s}$ for reperfusion. We performed data analysis using MATLAB-based DCE-US software. An artery in the same depth as the region of interest (ROI) was used for scaling. The measurements were compared using the intraclass correlation coefficient (ICC) and Bland Altman plots.

Results The interobserver agreement on the Logiq E9 for MTT (ICC $=0.83$, confidence interval $(\mathrm{Cl}) 0.46-0.96$ ) was excellent. There was poor agreement for MTT between the Logiq E9 and the IU22 (ICC $=-0.084, \mathrm{Cl}-0.68-0.58)$. The interobserver agreement for blood volume measurements was excellent on the Logiq E9 (ICC $=0.9286, \mathrm{Cl} 0.7250-0.98$ ) and between scanners (ICC $=0.86, \mathrm{Cl}=0.50-0.97)$.

Conclusion Interobserver agreement was excellent using the same scanner for both parameters and between scanners for $\mathrm{BV}$, but the comparison between two scanners did not yield acceptable agreement for MTT. This was probably due to incomplete bursting of bubbles in some of the recordings on the IU22. 


\section{Introduction}

Dynamic contrast-enhanced ultrasonography (DCE-US) imaging is a real-time, low-cost application that can be used to measure perfusion in tissue. An ultrasound contrast agent (UCA) is given intravenously and a selected area is observed over time. Ultrasound intensity in a region of interest (ROI) plotted over time results in a time intensity curve (TIC). Since ultrasound data are relatively noisy, it is common to fit the data to mathematical models from which a number of amplitude or temporal features can be derived [1,2].

DCE-US is not included in clinical guidelines $[3,4]$, which is most likely due to standardization difficulties $[5,6]$. Briefly summarized, differences in settings of the ultrasound scanner, patient characteristics, injection technique, ultrasound contrast behavior, presence of large vessels in the ROI, region selection and tissue motion are all factors that may introduce variability [5-8]. Studies with strict standardization criteria show promising results [9], while there is high variability and low reproducibility when attempts are made to compare different equipment [10].

The two main approaches for contrast administration when performing DCE-US are disruption replenishment [11] and bolus tracking [9]. With bolus tracking the evolution of tissue concentration is observed over time after a bolus injection of UCA. The method is easy to apply and allows the estimation of absolute perfusion parameters. Unfortunately, patient characteristics and injection technique influence the temporal-based parameters. These differences can be described by the arterial input function (AIF). The AIF is mixed with tissue function in TIC, however. This makes it challenging to measure and model the actual tissue perfusion or tissue residue function (TRF) $[12,13]$. In the burst replenishment technique, the UCA is given as a steady infusion, the UCA in the ROI is destroyed with a burst of ultrasound with a high mechanical index and tissue reperfusion is observed. This process can be done repeatedly and has good reproducibility. However, absolute perfusion parameters cannot be obtained [11].

Recently, a third approach was suggested by jiřík et al., proposing a new technique for ultrasound perfusion analysis: the bolusand-burst method in combination with a pharmacokinetic model based on the AIF and TRF [13]. This method combines bolus tracking with burst replenishment, i. e., a burst is applied when the tissue concentration has reached its plateau, and allows for the estimation of the AIF and the true mean transit time of the tissue $[13,14]$. The method has been further extended to use a fully parametric pharmacokinetic model [15]. It allows absolute quantification of the mean capillary transit time (MTT), blood volume (BV), and hence also blood flow (BF) using the central volume theorem $(\mathrm{BF}=\mathrm{BV} / \mathrm{MTT})$.

Absolute quantification in the bolus-and-burst method can be achieved by a scaling procedure using the TIC in a vessel (artery or vein). Absolute quantification can lead to a reduced variability of perfusion-parameter estimates caused by differences in ultrasound scanner type and settings $[6,13,16]$.

The bolus-and-burst method has been used to measure absolute blood flow in the intestine and pancreas [14, 17, 18]. Perfusion measurements with DCE-US could prove useful as a noninvasive technique for diagnosing chronic pancreatitis and the loss of exocrine function $[17,19]$. However, as we have previously demonstrated, perfusion imaging of the pancreas is challenging and com- bined with the complexity of the method this has implications for the interobserver agreement of measurements in the clinical application of the model (17). In the present study, the aim was to estimate interobserver agreement in a population with mixed pancreatic exocrine function using the same ultrasound scanner and agreement between two different scanner systems.

\section{Materials and Methods}

\section{Subjects}

During a one-year period (April 2014-April 2015), patients admitted to pancreatic ultrasound due to various pancreatic diseases or suspicion thereof were prospectively recruited to the study. As the study was designed to look at technical reproducibility between repeated injections by two investigators and on two ultrasound scanners, a preliminary B-mode scan of the pancreas was performed prior to DCE-US and subjects with insufficient visualization of the pancreas were not included. Also, patients were not included if both investigators were not available. No patients were excluded from the study after DCE-US had been performed. We included 10 patients with suspected chronic pancreatitis and cystic fibrosis with suspected exocrine failure. The subjects represent the whole range from severe pancreatic failure to a normal functioning pancreas.

The protocol was approved by the Regional Committee for Medical and Health Research Ethics in Western Norway (approval number: REK: 2010/2857-7) and the study was performed in accordance with the Helsinki Declaration. All subjects signed an informed consent form.

\section{Patient characterization}

Patient records were reviewed and all subjects were interviewed. The age and sex of the patient, body mass index, and final diagnosis were documented ( $\triangleright$ Table $\mathbf{1}$ ).

\section{Dynamic contrast-enhanced ultrasonography}

The subjects fasted $>4 \mathrm{~h}$ before the examination. Scanning of the pancreas was performed with the subjects in a supine position using a transverse or oblique epigastric probe position.

A GE Logic E9 scanner (GE Healthcare, Milwaukee, WI, USA) and an IU22 ultrasound scanner (Philips, Best, the Netherlands) and their corresponding curvilinear probes for abdominal use ( $C 1-5$ for Logiq E9 and C5-1 for IU22) were applied.

The general default contrast settings were used on each scanner and the method for contrast detection was amplitude modulation. Scanner settings such as gain, dynamic range of the video, color map and imaging depth remained constant throughout the study. On the Logiq E9 the send frequency of the transducer was $2.5 \mathrm{MHz}$, the mechanical index (MI) was 0.10 during DCE-US and 0.85 during a 10 -frame burst while the dynamic range was 66 and the color map was $2 / 0$. On the IU22 the send frequency of the transducer was $1.7 \mathrm{MHz}$, the MI was 0.05 during DCE-US and 0.75 during a 3 -frame burst while the dynamic range was 50 , the gray map was 2 and the chroma map was 3. Gain was adjusted to minimize background signal from the tissue and was thus adjusted for each study. 
- Table 1 Patient demographics in a study using ultrasound and contrast agents to improve pancreatic imaging.

\begin{tabular}{|c|c|c|c|c|c|}
\hline Patient & Diagnosis * & Gender $(F=$ female, $M=$ male $)$ & Age (years) & F-elastase $(\mu \mathrm{g} / \mathrm{g})$ & BMI $\left(\mathrm{kg} / \mathrm{m}^{2}\right)$ \\
\hline 1 & SCP & M & 25 & 125 & 21.8 \\
\hline 2 & SCP & $\mathrm{F}$ & 79 & 148 & 21.1 \\
\hline 3 & $\mathrm{CP}$ & M & 52 & 122 & 22.6 \\
\hline 4 & CF & $\mathrm{F}$ & 16 & N/A & 25.3 \\
\hline 5 & SCP & M & 76 & 146 & N/A \\
\hline 6 & $\mathrm{CP}$ & M & 59 & 95 & 24.2 \\
\hline 7 & $\mathrm{CF}$ & $\mathrm{F}$ & 40 & 42 & 29.0 \\
\hline 8 & $\mathrm{CP}$ & $\mathrm{F}$ & 78 & 83 & 22.9 \\
\hline 9 & $\mathrm{CP}$ & $\mathrm{F}$ & 39 & 66 & 22.7 \\
\hline 10 & $\mathrm{CF}$ & $\mathrm{F}$ & 56 & 419 & 17.0 \\
\hline
\end{tabular}

The first examination was performed on the Logiq E9 by observer 1 (TE). The second examination was performed on the same scanner by observer $2(\mathrm{KN})$. Observer 2 was blinded to the study performed by observer 1 and did not know the probe placement nor the imaging plane used by observer 1 . The third examination was performed on the IU22 by observer 1 who could not be blinded to his first examination on the Logiq $\mathrm{E} 9$. He aimed to reproduce the imaging planes between the two examinations. There was an interval of 10 min between the examinations, allowing the contrast to dissipate. For each examination $1.5 \mathrm{ml}$ of the ultrasound contrast agent SonoVue (Bracco, Milan, Italy) was given as a bolus over $2 \mathrm{~s}$ and flushed with $10 \mathrm{ml}$ of saline over $4 \mathrm{~s}$ through an IV line with a diameter of $1.1 \mathrm{~mm}$ placed in the left cubital fossa.

The examiner located a part of the pancreas with minimal movement and interference from air shadowing, also avoiding large vessels between the probe and the pancreas. A recording of the contrast enhancement was performed for $60 \mathrm{~s}$ before the burst and the scan continued for a further $30 \mathrm{~s}$. Thus, 90 -second video recordings were acquired for each bolus injection. The videos were exported in DICOM format, which could be uploaded to the DCE-US software.

\section{Perfusion analysis}

The DCE-US (http://www.isibrno.cz/perfusion/) software is MAT$\mathrm{LAB}$-based and structured according to the analysis process. To reduce the size of datasets and thus computational load, the sequences were down-sampled to about 3 frames per second. Subsequently, a manual movement correction and single frame exclusion procedure were performed as described in more detail in a previous paper [18]. After the manual correction, the video data was re-linearized using information from the color conversion map and the video dynamic range. Following the conversion from video to intensity-data linear to UCA concentration, an ROI was defined ( $>$ Fig. 1, panels a-c). The ROI was chosen in a region of the pancreas where movement and other artifacts were minimized. Finally, an artery in close relation to the ROI was identified ( $\triangleright$ Fig. 1, panels $d-f$ ) and the area under the TIC curve of the estimated AIF was equalized to the area under the curve of this artery for scaling [14].
The manual motion correction and perfusion analysis were performed by KN, who was blinded to the pancreatic function data and the genotype of the cystic fibrosis patients.

\section{Statistics}

Interobserver agreement for the parameters mean transit time and blood volume was examined using the intra-class correlation coefficient (ICC). Since blood flow is a product of these parameters, it was not included in the analysis of interobserver agreement. The ICC has values between 0 and 1 and is considered poor if $0-0.2$, fair if $0.2-0.4$, good if $0.4-0.75$ and excellent if $>0.75$. Interobserver variability was also examined with the Pearson correlation coefficient and the Bland Altman plot with limits of agreement. To test for fixed bias, a single sample T-test was performed, and to test for proportional bias, a linear regression analysis was performed. The data analysis was performed using IBM SPSS Statistics software version 23 (IBM Inc., Armonk, NY, USA).

\section{Results}

Patients with cystic fibrosis $(n=3)$, chronic pancreatitis $(n=4)$ and a normal pancreas $(n=3)$ were included. The patients represent the whole range from severe exocrine failure to a normal pancreas.

\section{Perfusion parameters}

Blood flow (BF) in mL/min/100 mL, blood volume (BV) in mL/100 mL and mean capillary transit time (MTT) in seconds were calculated in all subjects. The results are displayed in > Table 2 . One recording by the IU22 scanner was lost as it was not stored properly on the scanner.

There was excellent interobserver agreement for MTT on the Logiq E9 with an ICC of 0.83 (95\% confidence interval $(\mathrm{Cl}) 0.46$ 0.96). Between the Logiq $\mathrm{E} 9$ and the IU22, there was poor agreement for MTT $(\mathrm{ICC}=-0.084, \mathrm{Cl}-0.68-0.58)$. The interobserver agreement for blood volume measurements was excellent between observers on the Logiq E9 (ICC $=0.92, \mathrm{Cl} 0.72-0.98)$ and between scanners $(\mathrm{ICC}=0.86, \mathrm{Cl}=0.50-0.97)$. 

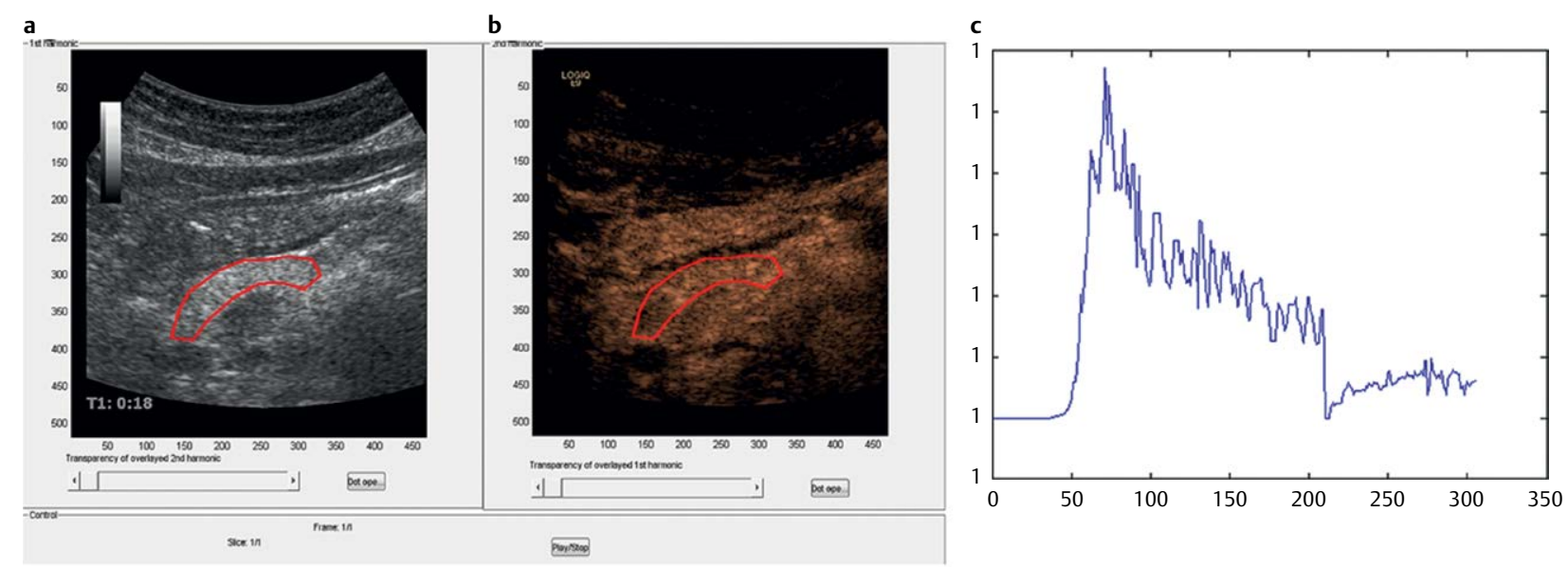

d
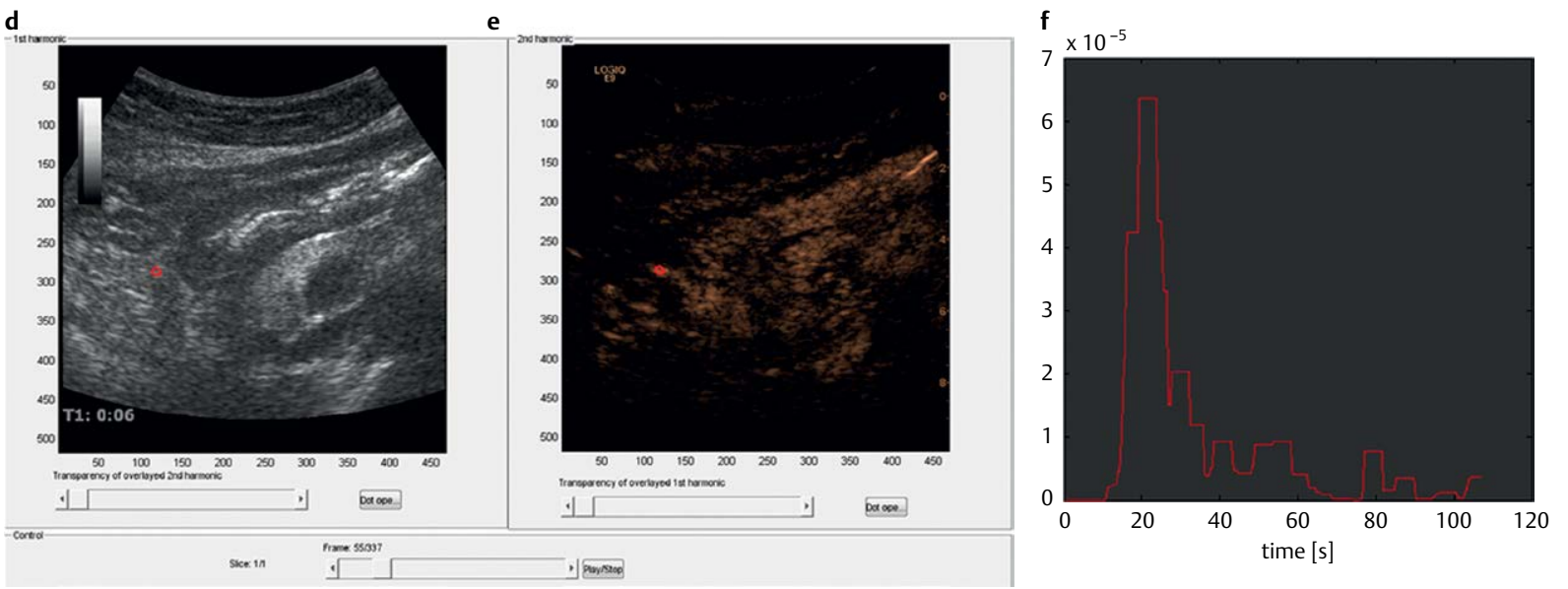

- Fig. 1 Panels a-c show the pancreatic B-mode image $\mathbf{a}$, contrast image $\mathbf{b}$ and the time intensity curve $\mathbf{c}$ of the region of interest (ROI, red). In panels $\mathbf{d}$-f the corresponding images are shown for an artery (in red) used for scaling the dataset in a study using ultrasound and contrast agents.

There was a significant correlation between observer 1 and observer 2 on the Logiq E9 for MTT $(p=0.001)$ and BV $(p=<0.001)$ with correlation coefficients of 0.87 and 0.93 , respectively ( $\triangleright$ Fig. 2 , panels a and c). For the comparison between the Logiq E9 and IU22 using the same examiner (TE), there was no correlation for the MTT, but significant correlation for blood volume $(r=0.88, p=0.002$,

- Fig. 2, panels b and d).

Bland Altman plots of the MTT and BV were drawn comparing recordings between operators ( $\vee$ Fig. 3 panel a and b,) and different scanners ( $\triangleright$ Fig. 3, panels c and d). There was no fixed or proportional bias between observer 1 and 2 on the Logiq E9. However, between the Logiq E9 and the IU22, there was a fixed bias for the blood volume calculation with the IU22 giving consistently lower values. There was also a fixed bias for the MTT.

\section{Discussion}

In this study, we found excellent interobserver agreement for repeated measurements of all perfusion parameters using the same ultrasound scanner with the bolus-and-burst method. Between the scanners used in this study, the interobserver agreement was excellent for blood volume, but poor for mean transit time.

The interobserver agreement of the bolus-and-burst method in our study is comparable to that of the burst replenishment technique as applied by Atri et al. [20]. They investigated tumors with variable vascularity, testing different approaches and measured ICC for blood volume estimates between 0.78 and 0.97 .

Another study by Gauthier et al. of the bolus method applied on a skin tumor model in nude mice demonstrated an intraobserver variability with a coefficient of variation between 9.06-15.79 for AUC from a time intensity curve [21]. However, this was an experimental study on mice with a strict setup, while our examinations were performed on patients in a clinical setting.

As mentioned in the introduction, only one study has previously addressed the variability of measurements between scanners [10]. They used the Vuebox software which analyzes time intensity curves. This software provides both amplitude-related variables, such as area under the curve, and time-related variables, such as mean transit time, rise time and fall time. This method does not correct for the arterial input function and has no internal scaling to 
- Table 2 Mean transit time (MTT, s), blood volume (BV, $\mathrm{ml} / 100 \mathrm{ml}$ tissue) and blood flow (BF, $\mathrm{ml} / \mathrm{min} / 100 \mathrm{ml}$ tissue) measurements for every patient in a study on the pancreas for the Logiq E9 (LE91- = observer 1, LE9-2 = observer 2) and the IU22 ultrasound scanner (IU22). The data from patient 4 on the IU22 was lost as it was not saved correctly on the ultrasound scanner.

\begin{tabular}{|c|c|c|c|c|c|c|c|c|c|c|}
\hline Patient & Diagnosis & $\begin{array}{c}\text { MTT } \\
\text { LE9-1 }\end{array}$ & $\begin{array}{c}\text { MTT } \\
\text { LE9-2 }\end{array}$ & $\begin{array}{l}\text { MTT } \\
\text { IU22 }\end{array}$ & $\begin{array}{c}\text { BV } \\
\text { LE9-1 }\end{array}$ & $\begin{array}{c}\text { BV } \\
\text { LE9-2 }\end{array}$ & $\begin{array}{c}\text { BV } \\
\text { IU22 }\end{array}$ & $\begin{array}{c}\text { BF } \\
\text { LE9-1 }\end{array}$ & $\begin{array}{c}\text { BF } \\
\text { LE9-2 }\end{array}$ & $\begin{array}{c}\text { BF } \\
\text { IU22 }\end{array}$ \\
\hline & & \multicolumn{3}{|c|}{ (seconds) } & \multicolumn{3}{|c|}{ (ml/100 ml tissue) } & \multicolumn{3}{|c|}{ (ml/min/100 ml tissue) } \\
\hline 1 & SCP & 4.0 & 3.5 & 3.4 & 2.6 & 3.0 & 1.2 & 38.9 & 51.0 & 20.62 \\
\hline 2 & SCP & 3.6 & 4.9 & 1.6 & 2.0 & 2.0 & 0.3 & 32.8 & 23.7 & 10.9 \\
\hline 3 & $\mathrm{CP}^{*}$ & 11.7 & 12.7 & 2.0 & 2.7 & 2.6 & 1.3 & 12.2 & 12.3 & 40.2 \\
\hline 4 & CF & 10.4 & 16.2 & NA & 1.9 & 1.8 & NA & 10.9 & 6.5 & NA \\
\hline 5 & $\mathrm{SCP} *$ & 7.3 & 7.5 & 1.2 & 2.5 & 2.1 & 2.6 & 20.7 & 16.6 & 124.9 \\
\hline 6 & $\mathrm{CP}$ & 5.3 & 5.5 & 5.8 & 1.8 & 2.2 & 1.2 & 20.2 & 24.1 & 12.6 \\
\hline 7 & CF & 3.9 & 3.7 & 3.3 & 2.7 & 2.6 & 2.4 & 41.0 & 41.8 & 43.2 \\
\hline 8 & $\mathrm{CP}^{*}$ & 10.6 & 8.4 & 1.5 & 4.9 & 4.3 & 4.2 & 28.0 & 30.6 & 166.3 \\
\hline 9 & $\mathrm{CP}$ & 8.8 & 8.2 & 8.5 & 1.7 & 1.3 & 1.0 & 11.5 & 9.4 & 6.7 \\
\hline 10 & CF & 5.0 & 4.4 & 4.9 & 3.2 & 3.0 & 3.1 & 38.0 & 41.6 & 38.0 \\
\hline
\end{tabular}

${ }^{*}$ In these patients there was residual tissue enhancement in the pancreas after the burst on the IU22 ultrasound scanner (SCP= suspected chronic pancreatitis, $\mathrm{CP}=$ chronic pancreatitis, $\mathrm{CF}=$ cystic fibrosis)
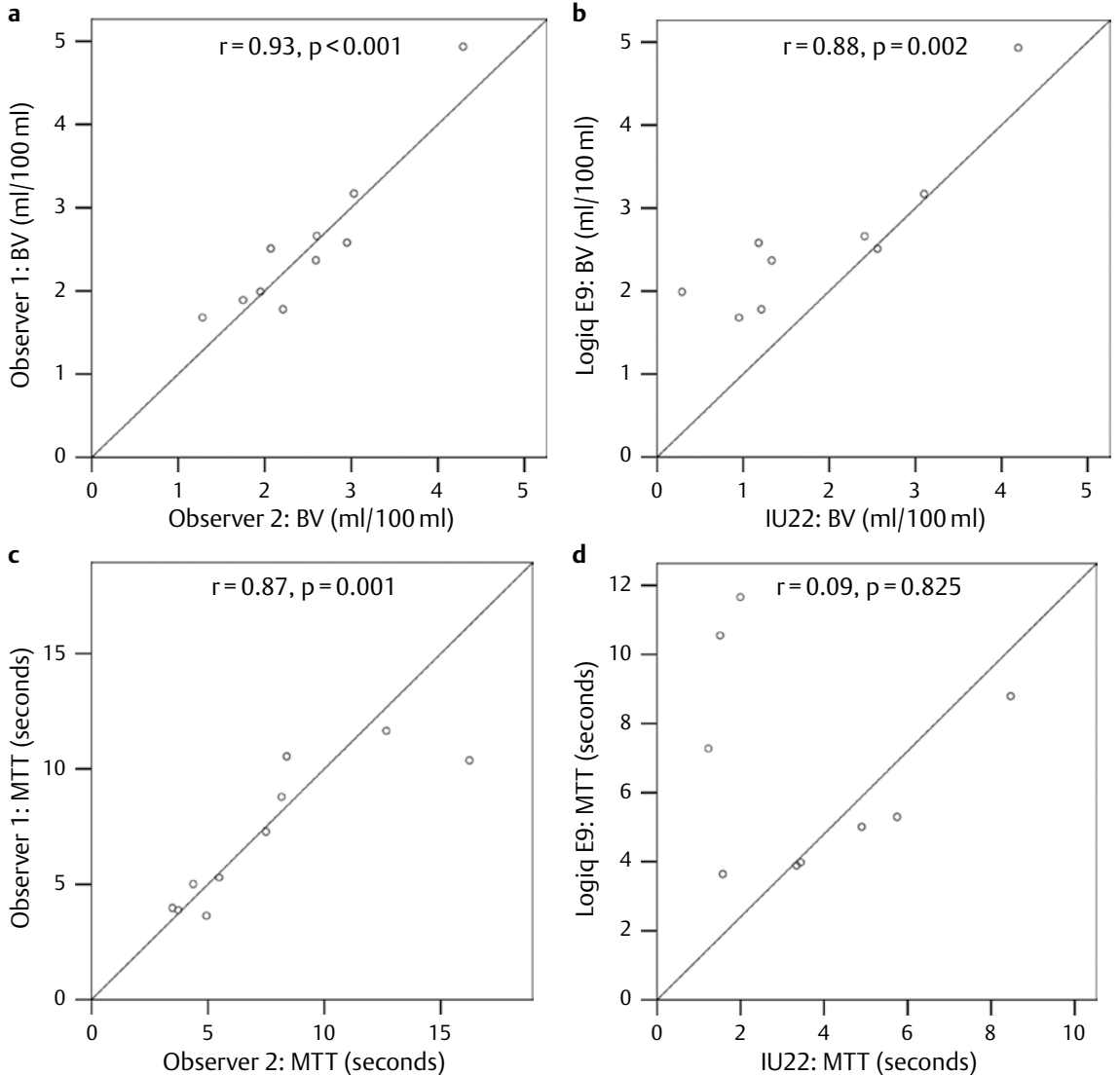

- Fig. 2 Panels $\mathbf{a}$ and $\mathbf{b}$ show correlations for blood volume (BV) between observer 1 and 2 on the Logiq E9 and between Logic E9 and IU22, respectively, in a study using ultrasound and contrast agents to improve pancreatic imaging. Panels $\mathbf{c}$ and $\mathbf{d}$ show the corresponding correlations for mean transit time (MTT). 

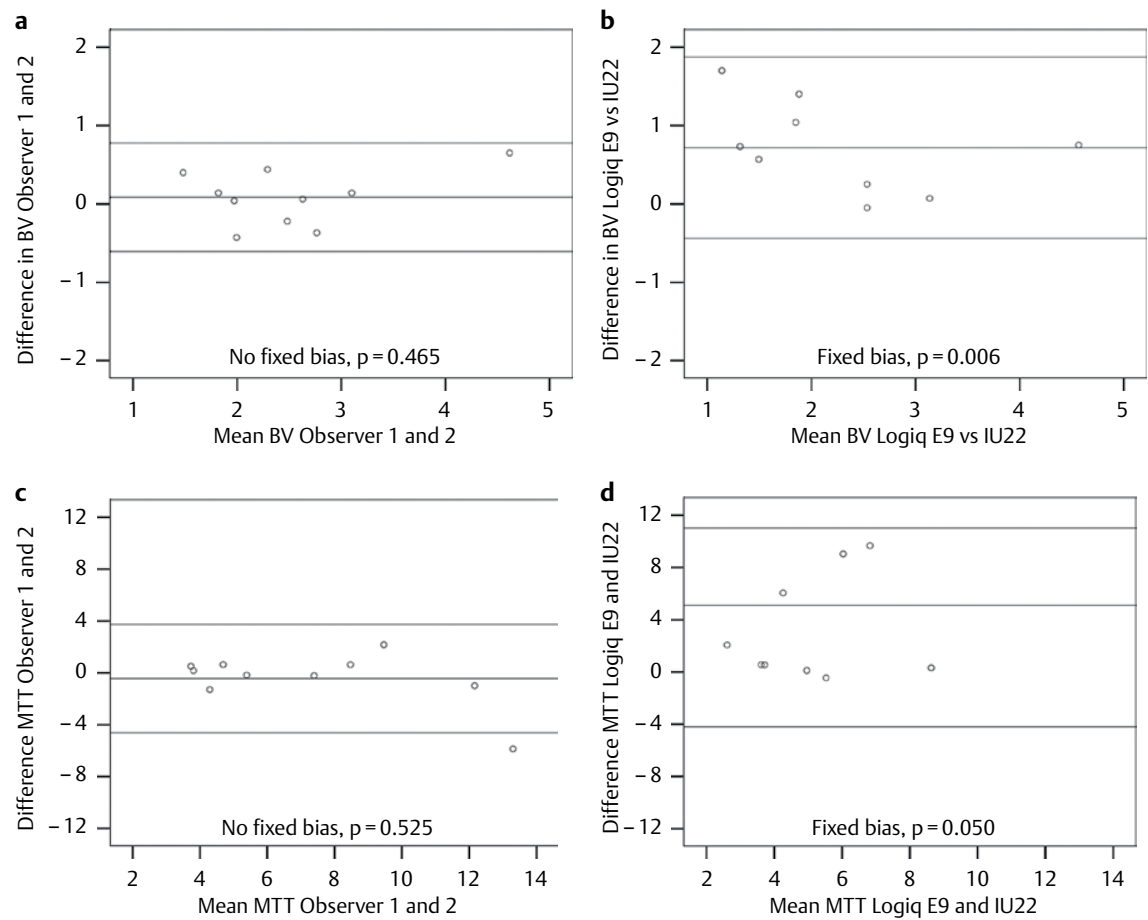

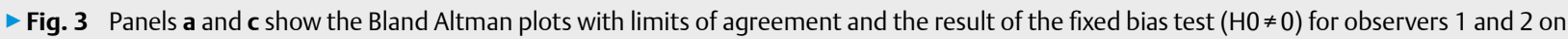
the Logiq E9 scanner for blood volume and mean transit time, respectively. Panels $\mathbf{b}$ and $\mathbf{d}$ show the corresponding Bland Altman plots for the Logiq E9 and the IU22 in a study using contrast agents to improve pancreatic imaging.

absolute values. Although the software in this study was calibrated to the settings of ultrasound probes and scanners, they did not achieve good interobserver agreement. In our study, the interobserver agreement for blood volume measurements between scanners was excellent although there was a fixed bias between the scanners. One explanation for such a bias could be differences in dynamic range between the scanners.

For the whole group of patients, there was no relationship between the MTTs measured on the IU22 and the Logiq E9. While going through recordings from the IU22, it became clear through visual inspection that for some patients $(n=3)$ the burst was not sufficient for clearing all the bubbles in the ROI. In these patients, the mean transit time was very short, reflecting this incomplete bursting. In the patients where the burst did remove all microbubbles (patients no. 1, 2, 6, 7, 9 and 10), there was good concordance between the IU22 and Logiq E9 ( $r=0.94, p=0.016)$.

The bolus-and-burst method with internal scaling could potentially solve many of the problems with variability in perfusion analysis as the MTT estimate is corrected using the AIF. Furthermore, the issues of scanner variability are reduced by internal scaling. Nevertheless, some standardization of scanner settings and contrast use are needed. The mechanical index should be low (0.05-0.19) so that bubble destruction is minimized and the dynamic range of the exported videos should be at least $45 \mathrm{~dB}$ to reduce errors in reconverting the videos to linear data [7, 22]. Also the focus should not be within the imaged region, but rather somewhat deeper as this reduces variability intensity with the imaged region [6].
The issue with sampling a 2D region in 3D tissue and out-ofplane motion was not addressed specifically in this paper. However, the interobserver agreement was excellent even though the second observer was blinded to the first observer's plane selection.

\section{Limitations of study}

The pancreas is located deep in the upper part of the abdomen and is frequently hidden behind air-containing organs such as the colon, the stomach or the duodenum. Consequently, there are challenges regarding CEUS recordings since the pancreas is situated relatively close to the diaphragm and abdominal aorta scanning will be disturbed by respiratory movements and pulsation [18, 23]. To deal with these challenges, we chose to include only patients with optimal scanning conditions judged by preliminary ultrasound scanning of the pancreas. However, this approach introduces a selection bias. We still argue that these measures were necessary to reduce the disturbances introduced by poor scanning quality in the final analysis. Putting quality requirements on scanning conditions is not rare in diagnostic decision making from sonographic imaging.

In scanning protocols where a burst is applied, this should be standardized to ensure that the bubbles on the imaging plane are sufficiently destroyed. For simplicity, the default settings for contrast detection were used in each scanner as were the settings for the burst. Upon analysis, it became apparent that the burst on the IU22 was not sufficient in some patients. In retrospect, we should have increased the burst length and MI on the IU22. 
The manual motion correction that was performed was cumbersome and time-consuming. A number of out-of-plane frames were excluded. This was done by the same investigator to reduce variability for this part of the analysis. Scaling was also challenging as it required the identification of an artery at the same depth as the ROI. Together, these two steps may account for some of the individual variations between the observers. Furthermore, the manual exclusion process may introduce selection bias in the analysis. In the future these steps should be automated to reduce user effects and improve the applicability of the technique.

Repeated contrast boluses may affect results since there could be leftover UCA bubbles in the circulation from the previous bolus. We tried to avoid this by waiting 5-10 min between the boluses and by removing the microbubbles from microcirculation by applying repeated high Ml bursts between the bolus injections. Upon inspection, there was no visible UCA in the ROI before the next bolus was given. We believe that this factor has minimal influence due to the fact that the perfusion calculations are adjusted to baseline noise before the arrival of the UCA in the ROI.

\section{Conclusion}

We find very good correlation and good repeatability with the same scanner system. Improvement of the software aiding adequate standardization and enhanced and simplified motion correction and scaling process may eliminate some of the observed variability. We have demonstrated that ultrasound imaging of the pancreas in general and contrast-enhanced ultrasound in particular pose challenges regarding image quality, reproducibility and standardization [17]. The process of movement correction and exclusion of image disturbances and the analysis itself in the bolus-and-burst software are manual and time-consuming and involve several steps that are likely to reduce interobserver agreement. Forthcoming software improvements to reduce the number of manual steps and to automatize tissue recognition are highly warranted to increase the clinical applicability of the method.

\section{Acknowledgements}

We are indebted to the personnel at the gastrolab at Haukeland University Hospital without whom this study could not have been performed.

\section{conflicts of interest}

Odd Helge Gilja: Lecture fees GE Healthcare, Takeda AS, MEDA AS Kim Nylund: Lecture fees MEDA AS, Ferring Pharmaceuticals
[1] Dietrich CF, Averkiou MA, Correas JM, Lassau N, Leen E, Piscaglia F. An EFSUMB introduction into Dynamic Contrast-Enhanced Ultrasound (DCE-US) for quantification of tumour perfusion. Ultraschall in der Medizin 2012; 33: 344-351

[2] Frohlich E, Muller R, Cui XW, Schreiber-Dietrich D, Dietrich CF. Dynamic contrast-enhanced ultrasound for quantification of tissue perfusion. Journal of ultrasound in medicine: Official journal of the American Institute of Ultrasound in Medicine 2015; 34: 179-196

[3] Claudon M, Dietrich CF, Choi BI, Cosgrove DO, Kudo M, Nolsoe CP et al. Guidelines and good clinical practice recommendations for Contrast Enhanced Ultrasound (CEUS) in the liver - update 2012: A WFUMBEFSUMB initiative in cooperation with representatives of AFSUMB, AIUM, ASUM, FLAUS and ICUS. Ultrasound in Medicine \& Biology 2013; 39: $187-210$

[4] Piscaglia F, Nolsoe C, Dietrich CF, Cosgrove DO, Gilja OH, Bachmann Nielsen $\mathrm{M}$ et al. The EFSUMB Guidelines and Recommendations on the Clinical Practice of Contrast Enhanced Ultrasound (CEUS): update 2011 on non-hepatic applications. Ultraschall in der Medizin. 2012; 33: 33-59

[5] Hudson JM, Williams R, Tremblay-Darveau C, Sheeran PS, Milot L, Bjarnason GA et al. Dynamic contrast enhanced ultrasound for therapy monitoring. European journal of radiology 2015; 84: 1650-1657

[6] Tang MX, Mulvana H, Gauthier T, Lim AK, Cosgrove DO, Eckersley RJ et al. Quantitative contrast-enhanced ultrasound imaging: A review of sources of variability. Interface focus 2011; 1: 520-539

[7] Gauthier TP, Averkiou MA, Leen EL. Perfusion quantification using dynamic contrast-enhanced ultrasound: the impact of dynamic range and gain on time-intensity curves. Ultrasonics 2011; 51: 102-106

[8] Gauthier TP, Chebil M, Peronneau P, Lassau N. In vitro evaluation of the impact of ultrasound scanner settings and contrast bolus volume on time-intensity curves. Ultrasonics 2012; 52: 12-19

[9] Lassau N, Bonastre J, Kind M, Vilgrain V, Lacroix J, Cuinet M et al. Validation of dynamic contrast-enhanced ultrasound in predicting outcomes of antiangiogenic therapy for solid tumors: The French multicenter support for innovative and expensive techniques study. Investigative radiology 2014; 49: 794-800

[10] Zink F, Kratzer W, Schmidt S, Oeztuerk S, Mason RA, Porzner M et al. Comparison of two high-end ultrasound systems for contrastenhanced ultrasound quantification of mural microvascularity in crohn's disease. Ultraschall in der Medizin 2016; 37: 74-81

[11] Hudson JM, Leung K, Burns PN. The lognormal perfusion model for disruption replenishment measurements of blood flow: In vivo validation. Ultrasound in medicine \& biology 2011; 37: 1571-1578

[12] Gauthier M, Tabarout F, Leguerney I, Polrot M, Pitre S, Peronneau P et al. Assessment of quantitative perfusion parameters by dynamic contrast-enhanced sonography using a deconvolution method: An in vitro and in vivo study. Journal of ultrasound in medicine: official journal of the American Institute of Ultrasound in Medicine 2012; 31: 595-608

[13] Jirik R, Nylund K, Gilja OH, Mezl M, Harabis V, Kolar R et al. Ultrasound perfusion analysis combining bolus-tracking and burst-replenishment. IEEE transactions on ultrasonics, ferroelectrics, and frequency control 2013; 60: 310-319

[14] Nylund K, Jirik R, Mezl M, Leh S, Hausken T, Pfeffer F et al. Quantitative contrast-enhanced ultrasound comparison between inflammatory and fibrotic lesions in patients with Crohn's disease. Ultrasound in Medicine \& Biology 2013; 39: 1197-1206 
[15] Jirik RNK, Taxt T, Mezl Martin, Hausken T, Harabis V, Kolar R, Standara $\mathrm{M}$, Gilja OH. Parametric ultrasound perfusion analysis combining bolus tracking and replenishment 2012 IEEE International Ultrasonics Symposium: IEEE. 2012; 1323-1326

[16] Jirik R, Soucek K, Mezl M, Bartos M, Drazanova E, Drafi F et al. Blind deconvolution in dynamic contrast-enhanced MRI and ultrasound. Conference proceedings: Annual International Conference of the IEEE Engineering in Medicine and Biology Society IEEE Engineering in Medicine and Biology Society Annual Conference 2014; 2014: 4276-4279

[17] Engjom TLB, Nylund K, Erchinger F, Gilja OH, Dimcevski G. 229 Pancreatic genotype-phenotype co-variations in cystic fibrosis. Journal of Cystic Fibrosis 2015

[18] Schafer S, Nylund K, Saevik F, Engjom T, Mezl M, Jirik R et al. Semi-automatic motion compensation of contrast-enhanced ultrasound images from abdominal organs for perfusion analysis. Computers in biology and medicine 2015; 63: 229-237

[19] Lewis MP, Lo SK, Reber PU, Patel A, Gloor B, Todd KE et al. Endoscopic measurement of pancreatic tissue perfusion in patients with chronic pancreatitis and control patients. Gastrointestinal endoscopy 2000; 51: 195-199
[20] Atri M, Hudson JM, Sinaei M, Williams R, Milot L, Moshonov H et al. Impact of acquisition method and region of interest placement on inter-observer agreement and measurement of tumor response to targeted therapy using dynamic contrast-enhanced ultrasound. Ultrasound in Medicine \& Biology 2016; 42: 763-768

[21] Gauthier M, Leguerney I, Thalmensi J, Chebil M, Parisot S, Peronneau P et al. Estimation of intra-operator variability in perfusion parameter measurements using DCE-US. World Journal of Radiology 2011; 3: $70-81$

[22] Rognin NG, Frinking P, Costa M, Arditi M.editors. In-vivo perfusion quantification by contrast ultrasound: Validation of the use of linearized video data vs. raw RF data. 2008 IEEE Ultrasonics Symposium; 2008 2-5 Nov. 2008;

[23] Erchinger F, Dimcevski G, Engjom T, Gilja OH. Transabdominal ultrasonography of the pancreas: basic and new aspects. Imaging in Medicine 2011; 3: 11 